\title{
Double point self-intersection surfaces of immersions
}

\author{
Mohammad A Asadi-Golmankhaneh \\ Peter J ECCles \\ Department of Mathematics, University of Urmia \\ PO Box 165, Urmia, Iran \\ and \\ Department of Mathematics, University of Manchester \\ Manchester, M13 9PL, UK \\ Email: pjeccles@man.ac.uk
}

\begin{abstract}
A self-transverse immersion of a smooth manifold $M^{k+2}$ in $\mathbb{R}^{2 k+2}$ has a double point self-intersection set which is the image of an immersion of a smooth surface, the double point self-intersection surface. We prove that this surface may have odd Euler characteristic if and only if $k \equiv 1 \bmod 4$ or $k+1$ is a power of 2. This corrects a previously published result by András Szücs [22].

The method of proof is to evaluate the Stiefel-Whitney numbers of the double point self-intersection surface. By the methods of [2] these numbers can be read off from the Hurewicz image $h(\alpha) \in H_{2 k+2} \Omega^{\infty} \Sigma^{\infty} M O(k)$ of the element $\alpha \in \pi_{2 k+2} \Omega^{\infty} \Sigma^{\infty} M O(k)$ corresponding to the immersion under the PontrjaginThom construction.
\end{abstract}

AMS Classification numbers Primary: 57R42

Secondary: 55R40, 55Q25, 57R75

Keywords: immersion, Hurewicz homomorphism, spherical class, Hopf invariant, Stiefel-Whitney number

Proposed: Ralph Cohen

Seconded: Gunnar Carlsson, Yasha Eliashberg
Received: 30 July 1999

Accepted: 29 February 2000

\section{Copyright Geometry and Topology}




\section{Introduction}

Let $f: M^{n-k} \uparrow \rightarrow \mathbb{R}^{n}$ be a self-transverse immersion of a compact closed smooth $(n-k)$-dimensional manifold in $n$-dimensional Euclidean space $(0<k \leq n)$. A point of $\mathbb{R}^{n}$ is an $r$-fold self-intersection point of the immersion if it is the image under $f$ of (at least) $r$ distinct points of the manifold. The selftransversality of $f$ implies that the set of $r$-fold self-intersection points (the $r$-fold self-intersection set) is itself the image of an immersion

$$
\theta_{r}(f): \Delta_{r}(f) \leftrightarrow \mathbb{R}^{n}
$$

of a compact manifold $\Delta_{r}(f)$, the $r$-fold self-intersection manifold, of dimension $n-r k$, although in general this immersion will not be self-transverse. It is natural to ask what can be said about this $r$-fold intersection manifold: which manifolds can arise for each value of $n, k$ and $r$ ?

The simplest case is when $n=r k$ so that the self-intersection manifold is a finite set of points and this case was the first to be considered in detail (see [3], [10], [11], [12], [16]).

More recently cases of higher dimensional self-intersection manifolds have been considered. András Szücs was one of the first to do so and in [22] considered the simplest case of interest, when the double point self-intersection manifold is a surface; this arises for $n=2 k+2$. In this paper we return to this case using different methods to Szücs. Our main result is the following.

Theorem 1.1 For $k \geq 1$, there exists an immersion $f: M^{k+2} \rightarrow \mathbb{R}^{2 k+2}$ with double point self-intersection manifold of odd Euler characteristic if and only if $k \equiv 1 \bmod 4$ or $k+1$ is a power of 2 .

This result should be contrasted with Szücs' result which asserted that double point self-intersection manifolds of odd Euler characteristic can occur only if $k \equiv 1 \bmod 4$. Szücs's approach used differential topology and the argument in the case $k \equiv 3 \bmod 4$ was particularly delicate.

Our approach uses algebraic topology and in particular the correspondence between bordism groups and homotopy groups of Thom complexes. In [2] we described a general approach to these problems which gives a method for determining the bordism class of the self-intersection manifolds of any immersion: the unoriented bordism class of a manifold is determined by its Stiefel-Whitney numbers and the Stiefel-Whitney numbers of the self-intersection manifolds of an immersion can be read off from certain homological information about the 
immersion. For double point self-intersection surfaces the situation is particularly simple since there are only two bordism classes: a compact surface is a boundary if its Euler characteristic is even and is is a non-boundary bordant to the projective plane if its Euler characteristic is odd.

The paper is organized as follows. In Section 2 we recall the results needed from [2], establish our basic notation and outline the proof of the theorem covering the steps which apply in all cases. In Section 3 we complete the proof in the easiest case of $k$ even and this is followed by the proofs for $k \equiv 1 \bmod 4$ in Section 4 and $k \equiv 3 \bmod 4$ in Section 5. Finally, in Section 6, we comment on the relationship between our results and those in Szücs' paper [22]. Almost everything in that paper is confirmed by our methods.

Acknowledgements Most of the results in this paper are contained in the first author's thesis [1] which also contains other applications of these methods. He was supported by the University of Urmia and the Ministry of Culture and Higher Education of the Islamic Replublic of Iran during his time as a student at the University of Manchester.

\section{The Stiefel Whitney characteristic numbers of the double point self-intersection manifold}

Let $\operatorname{Imm}(n-k, k)$ denote the group of bordism classes of immersions $M^{n-k} \rightarrow$ $\mathbb{R}^{n}$ of compact closed smooth manifolds in Euclidean $n$-space. Details of (co)bordism in this setting have been given by $\mathrm{R}$ Wells in [24]. By general position every immersion is regularly homotopic (and so bordant) to a self-transverse immersion and so each element of $\operatorname{Imm}(n-k, k)$ can be represented by a self-transverse immersion. In the same way bordisms between self-transverse immersions can be taken to be self-transverse; it is clear that such a bordism will induce a bordism of the immersions of the double point selfintersection manifolds so that $f \mapsto \theta_{2}(f)$ induces a double point self-intersection map

$$
\theta_{2}: \operatorname{Imm}(n-k, k) \rightarrow \operatorname{Imm}(n-2 k, 2 k) .
$$

Wells shows how $\operatorname{Imm}(n-k, k)$ may be described as a stable homotopy group. Let $M O(k)$ denote the Thom complex of a universal $O(k)$-bundle $\gamma^{k}: E O(k) \rightarrow$ $B O(k)$ (see [18] for basic material on vector bundles, Thom complexes and bordism theory). Using the Pontrjagin-Thom construction, Wells describes an isomorphism

$$
\phi: \operatorname{Imm}(n-k, k) \cong \pi_{n}^{S} M O(k) .
$$


Write $Q X$ for the direct limit $\Omega^{\infty} \Sigma^{\infty} X=\lim \Omega^{n} \Sigma^{n} X$, where $\Sigma$ denotes the reduced suspension functor and $\Omega$ denotes the loop space functor. By the adjointness of the functors $\Sigma$ and $\Omega, \pi_{n}^{S} M O(k) \cong \pi_{n} Q M O(k)$. We consider the $\mathbb{Z} / 2$-homology Hurewicz homomorphism

$$
h: \pi_{n}^{S} M O(k) \cong \pi_{n} Q M O(k) \rightarrow H_{n} Q M O(k)=H_{n}(Q M O(k) ; \mathbb{Z} / 2) .
$$

(Throughout this paper we use $H_{*}$ and $H^{*}$ to denote homology and cohomology with $\mathbb{Z} / 2$ coefficients.)

The main result of [2] describes how, for a self-transverse immersion $f: M^{n-k} \rightarrow$ $\mathbb{R}^{n}$ corresponding to $\alpha \in \pi_{n}^{S} M O(k)$, the Hurewicz image $h(\alpha) \in H_{n} Q M O(k)$ determines the normal Stiefel-Whitney numbers of the self-intersection manifolds $\Delta_{r}(f)$.

To state this result in the case of double point self-intersection manifolds we need some preliminaries.

The quadratic construction on a pointed space $X$ is defined to be

$$
D_{2} X=X \wedge X \rtimes_{\mathbb{Z} / 2} S^{\infty}=X \wedge X \times_{\mathbb{Z} / 2} S^{\infty} /\{*\} \times_{\mathbb{Z} / 2} S^{\infty},
$$

where the non-trivial element of the group $\mathbb{Z} / 2$ acts on $X \wedge X$ by permuting the co-ordinates and on the infinite sphere $S^{\infty}$ by the antipodal action. There is a natural map

$$
h^{2}: Q X \rightarrow Q D_{2} X
$$

known as a stable James-Hopf map which induces a stable Hopf invariant

$$
h_{*}^{2}: \pi_{n}^{S} X \rightarrow \pi_{n}^{S} D_{2} X
$$

(see [4] and [19]).

If the self-transverse immersion $f: M^{n-k} \rightarrow \mathbb{R}^{n}$ corresponds to the element $\alpha \in$ $\pi_{n}^{S} M O(k)$, then the immersion of the double point self-intersection manifold $\theta_{2}(f): \Delta_{2}(f) \rightarrow \mathbb{R}^{n}$ corresponds to the element $h_{*}^{2}(\alpha) \in \pi_{n}^{S} D_{2} M O(k)$ given by the stable Hopf invariant (see [15], [20], [21], [23]).

The immersion $\theta_{2}(f)$ corresponds to an element in the stable homotopy of $D_{2} M O(k)$ because the immersion of the double point self-intersection manifold automatically acquires additional structure on its normal bundle, namely that at each point $f\left(x_{1}\right)=f\left(x_{2}\right)$ the normal $2 k$-dimensional space is decomposed as the direct sum of the two (unordered) $k$-dimensional normal spaces of $f$ at the points $x_{1}$ and $x_{2}$. The universal bundle for this structure is

$$
\gamma^{k} \times \gamma^{k} \times_{\mathbb{Z} / 2} 1: E O(k) \times E O(k) \times_{\mathbb{Z} / 2} S^{\infty} \rightarrow B O(k) \times B O(k) \times_{\mathbb{Z} / 2} S^{\infty}
$$

which has the Thom complex $D_{2} M O(k)$. 
Forgetting this additional structure on the immersion corresponds to applying the map

$$
\xi_{*}: \pi_{n}^{S} D_{2} M O(k) \rightarrow \pi_{n}^{S} M O(2 k)
$$

induced by the map of Thom complexes $\xi: D_{2} M O(k) \rightarrow M O(2 k)$ which comes from the map $B O(k) \times B O(k) \times_{\mathbb{Z} / 2} S^{\infty} \rightarrow B O(2 k)$ classifying the bundle $\gamma^{k} \times \gamma^{k} \times_{\mathbb{Z} / 2} 1$. Thus, we have the following commutative diagram.

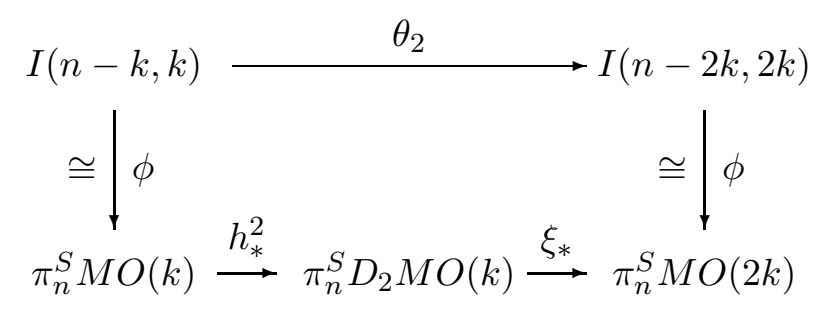

Turning now to homology, observe that, by adjointness, the stable James-Hopf map $h^{2}: Q X \rightarrow Q D_{2} X$ gives a stable map $\Sigma^{\infty} Q X \rightarrow \Sigma^{\infty} D_{2} X$ inducing a map in homology $h_{*}^{2}: H_{n} Q X \rightarrow H_{n} D_{2} X$. This gives the following commutative diagram.

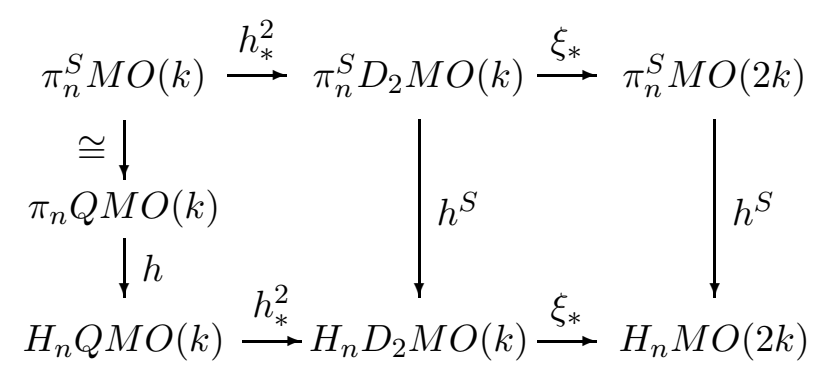

In this diagram the second and third vertical maps are stable Hurewicz homormorphisms defined using the fact the Hurewicz homomorphisms commute with suspension. The first square commutes by the definition of the stable Hurewicz map and by naturality, and the second square commutes by naturality.

Notice that the normal Stiefel-Whitney numbers (and so the bordism class) of the double point self-intersection manifold $\Delta_{2}(f)$ of an immersion $f: M^{n-k} \rightarrow$ $\mathbb{R}^{n}$ corresponding to $\alpha \in \pi_{n}^{S} M O(k)$ are determined by (and determine) the Hurewicz image $h^{S}(\beta)$ of the element $\beta=\xi_{*} h_{*}^{2}(\alpha) \in \pi_{n}^{S} M O(2 k)$ corresponding to the immersion $\theta_{2}(f)$. To be more explicit in the case under consideration we recall the structure of $H_{*} M O(k)$. 
Let $e_{i} \in H_{i} B O(1) \cong \mathbb{Z} / 2$ be the non-zero element (for $i \geq 0$ ). For each sequence $I=\left(i_{1}, i_{2}, \ldots, i_{k}\right)$ of non-negative integers we define

$$
e_{I}=e_{i_{1}} e_{i_{2}} \ldots e_{i_{k}}=\left(\mu_{k}\right)_{*}\left(e_{i_{1}} \otimes e_{i_{2}} \otimes \ldots e_{i_{k}}\right) \in H_{*} B O(k)
$$

where $\mu_{k}: B O(1)^{k} \rightarrow B O(k)$ is the map which classifies the product of the universal line bundles. The dimension of $e_{I}$ is $|I|=i_{1}+i_{2}+\ldots+i_{k}$.

From the definition of $\mu_{k}, e_{i_{1}} e_{i_{2}} \ldots e_{i_{k}}=e_{i_{\sigma(1)}} e_{i_{\sigma(2)}} \ldots e_{i_{\sigma(k)}}$ for each $\sigma \in \Sigma_{k}$, where $\Sigma_{k}$ is the permutation group on $k$ elements. Thus each such element can be written as $e_{i_{1}} e_{i_{2}} \ldots e_{i_{k}}$ where $i_{1} \leq i_{2} \leq \ldots \leq i_{k}$ and it follows by a counting argument that

$$
\left\{e_{i_{1}} e_{i_{2}} \ldots e_{i_{k}} \mid 0 \leq i_{1} \leq i_{2} \leq \ldots \leq i_{k}\right\}
$$

is a basis for $H_{*} B O(k)$ (see [14] Proposition 2.4.3).

The sphere bundle of the universal $O(k)$-bundle $\gamma^{k}$ is given up to homotopy by the inclusion $B O(k-1) \rightarrow B O(k)$ and so the Thom complex $M O(k)$ is homotopy equivalent to the quotient space $B O(k) / B O(k-1)$. It follows that

$$
\left\{e_{i_{1}} e_{i_{2}} \ldots e_{i_{k}} \mid 1 \leq i_{1} \leq i_{2} \leq \ldots \leq i_{k}\right\}
$$

is a basis for $\tilde{H}_{*} M O(k)$.

By Diagrams (1) and (2), the double point self-intersection surface of an immersion $M^{k+2} \rightarrow \mathbb{R}^{2 k+2}$ may be identified up to bordism by using the stable Hurewicz homomorphism

$$
h^{S}: \pi_{2 k+2}^{S} M O(2 k) \rightarrow H_{2 k+2} M O(2 k) .
$$

From the above, $H_{2 k+2} M O(2 k)$ has a basis $\left\{e_{1}^{2 k-1} e_{3}, e_{1}^{2 k-2} e_{2}^{2}\right\}$. The element $\beta \in \pi_{2 k+2}^{S} M O(2 k)$ corresponds to an immersion of a non-boundary (ie, a surface of odd Euler characteristic, bordant to the real projective plane) if and only if $h^{S}(\beta)=e_{1}^{2 k-1} e_{3}$, the only non-zero stably spherical element. This corresponds to the fact that a surface $L$ is a non-boundary if and only if the normal StiefelWhitney number $\bar{w}_{1}^{2}[L]=1$ (see [2] Proposition 3.4).

For $k>1$ we are in the stable range, $\pi_{2 k+2}^{S} M O(2 k) \cong \mathbb{Z} / 2$, and $h^{S}$ is a monomorphism: any two immersions of bordant manifolds are bordant. On the other hand, for $k=1$ the group $\operatorname{Imm}(2,4) \cong \pi_{4}^{S} M O(2)$ is infinite and $h^{S}$ is not a monomorphism. The bordism class of an immersion $L^{2} \rightarrow \mathbb{R}^{4}$ is not determined by $L$.

This discussion can be summed up in the following theorem which follows essentially from Diagrams (1) and (2). 
Theorem 2.1 Suppose that $f: M^{k+2} \rightarrow \mathbb{R}^{2 k+2}$ is a self-transverse immersion corresponding to $\alpha \in \pi_{2 k+2}^{S} M O(k)$. Then the double point self-intersection surface $\Delta_{2}(f)$ has odd Euler characteristic and so is a non-boundary if and only if

$$
\xi_{*} h_{*}^{2} h(\alpha)=e_{1}^{2 k-1} e_{3} \in H_{2 k+2} M O(2 k) .
$$

The map $h_{*}^{2}: H_{n} Q M O(k) \rightarrow H_{n} D_{2} M O(k)$ in Diagram (2) is very easy to describe in terms of the description of $H_{*} Q X$ as a Pontrjagin ring provided by Dyer and Lashof (see [9] or [17]). They make use of the Kudo-Araki operations $Q^{i}: H_{m} Q X \rightarrow H_{m+i} Q X$. These are trivial for $i<m$ and equal to the Pontrjagin square for $i=m$. If $I$ denotes the sequence $\left(i_{1}, i_{2}, \ldots, i_{r}\right)$ then we write $Q^{I} x=Q^{i_{1}} Q^{i_{2}} \ldots Q^{i_{r}} x$. The sequence $I$ is admissible if $i_{j} \leq i_{j+1}$ for $1 \leq j<r$ and its excess is given by $e(I)=i_{1}-i_{2}-\ldots-i_{r}$. With this notation we can give the description of $H_{*} Q X$ as a polynomial algebra: if $\left\{x_{\lambda} \mid \lambda \in \Lambda\right\}$ is a homogeneous basis for $\tilde{H}_{*} X \subseteq H_{*} Q X$ where $X$ is a path-connected space then

$$
H_{*} Q X=\mathbb{Z} / 2\left[Q^{I} x_{\lambda} \mid \lambda \in \Lambda, I \text { admissible of excess } e(I)>\operatorname{dim} x_{\lambda}\right] .
$$

Thus a basis for $H_{*} Q X$ is provided by the monomials in the polynomial generators.

We may define a height function ht on the monomial generators of $H_{*} Q X$ by $\operatorname{ht}\left(x_{\lambda}\right)=1, \operatorname{ht}\left(Q^{i} u\right)=2 \operatorname{ht}(u)$ and ht $(u \cdot v)=\operatorname{ht}(u)+\operatorname{ht}(v)$ (where $u \cdot v$ represents the Pontrjagin product). The following is a special case of Lemma 2.3 in [2].

Lemma 2.2 The homomorphism $h_{*}^{2}: \tilde{H}_{*} Q X \rightarrow \tilde{H}_{*} D_{2} X$ is given by projection onto the monomial generators of height 2. The kernel is spanned by the set of monomials of height other than 2 .

Corollary 2.3 A basis for $H_{2 k+2} D_{2} M O(k)$ is given by the following set:

$$
\left\{e_{1}^{k} \cdot e_{1}^{k-1} e_{3}, e_{1}^{k} \cdot e_{1}^{k-2} e_{2}^{2}, e_{1}^{k-1} e_{2} \cdot e_{1}^{k-1} e_{2}, Q^{k+2} e_{1}^{k}\right\}
$$

The map $\xi_{*}: H_{n} D_{2} M O(k) \rightarrow H_{n} M O(2 k)$ of Diagram (2) is also determined in [2]. In the case of $n=2 k+2$, Theorem 3.1 of [2] gives the following.

Lemma 2.4 The homomorphism $\xi_{*}: H_{2 k+2} D_{2} M O(k) \rightarrow H_{2 k+2} M O(2 k)$ is determined by the following values:

$$
\begin{aligned}
& \xi_{*}\left(e_{1}^{k} \cdot e_{1}^{k-1} e_{3}\right)=e_{1}^{2 k-1} e_{3} ; \\
& \xi_{*}\left(e_{1}^{k} \cdot e_{1}^{k-2} e_{2}^{2}\right)=\xi_{*}\left(e_{1}^{k-1} e_{2} \cdot e_{1}^{k-1} e_{2}\right)=e_{1}^{2 k-2} e_{2}^{2} ;
\end{aligned}
$$




$$
\xi_{*}\left(Q^{k+2} e_{1}^{k}\right)= \begin{cases}0 & \text { for } k \equiv 0 \bmod 4 \\ e_{1}^{2 k-1} e_{3} & \text { for } k \equiv 1 \bmod 4 \\ e_{1}^{2 k-2} e_{2}^{2} & \text { for } k \equiv 2 \bmod 4 \\ e_{1}^{2 k-1} e_{3}+e_{1}^{2 k-2} e_{2}^{2} & \text { for } k \equiv 3 \bmod 4\end{cases}
$$

Proof The first three results are immediate from [2] Theorem 3.1.

For the fourth we apply the formula given in the theorem:

$$
\begin{aligned}
& \xi_{*}\left(Q^{k+2} e_{1}^{k}\right)=\sum_{m_{1}+m_{2}+\ldots+m_{k}=2} \prod_{j=1}^{k}\left(\begin{array}{c}
m_{j}-1 \\
0
\end{array}\right) e_{1} e_{m_{j}+1} \quad\left(m_{j} \geq 0\right) \\
& =\sum_{m_{1}+m_{2}+\ldots+m_{k}=2} e_{1} e_{m_{1}+1} e_{1} e_{m_{2}+1} \ldots e_{1} e_{m_{k}+1} \quad\left(m_{j} \geq 0\right) \\
& =\left(\begin{array}{l}
k \\
1
\end{array}\right) e_{1}^{2 k-1} e_{3}+\left(\begin{array}{l}
k \\
2
\end{array}\right) e_{1}^{2 k-2} e_{2}^{2}
\end{aligned}
$$

which gives the required result.

To prove Theorem 1.1 using Theorem 2.1 we determine the image of the spherical classes in $H_{2 k+2} Q M O(k)$, ie, the classes in the image of $h: \pi_{2 k+2} Q M O(k) \rightarrow$ $H_{2 k+2} Q M O(k)$, under $\xi_{*} \circ h_{*}^{2}$ (see Diagram (2)). A complete description of these spherical classes is not necessary for it is sufficient to observe the following well-known properties of spherical classes (which are immediate from $H_{*} S^{n}$ by naturality).

Lemma 2.5 (a) If an homology class $u \in H_{n} X$ is spherical then it is primitive with respect to the cup coproduct, ie,

$$
\psi(u)=u \otimes 1+1 \otimes u,
$$

where $\psi: H_{n} X \rightarrow H_{n}(X \times X) \cong \sum_{i} H_{i} X \otimes H_{n-i} X$ is the map induced by the diagonal map.

(b) If an homology class $u \in H_{n} X$ is spherical (or stably spherical, ie, in the image of $\left.h^{S}: \pi_{n}^{S} X \rightarrow H_{n} X\right)$ then it is annihilated by the reduced Steenrod algebra, ie,

$$
S q_{*}^{i}(u)=0
$$

for all $i>0$, where $S q_{*}^{i}: H_{n} X \rightarrow H_{n-i} X$ is the vector space dual of the usual Steenrod square cohomology operation $S q^{i}: H^{n-i} X \rightarrow H^{n} X$.

To apply the first of these observations we determine the image of the coproduct primitive submodule of $\mathrm{H}_{2 k+2} Q M O(k)$ in $\mathrm{H}_{2 k+2} \mathrm{D}_{2} \mathrm{MO}(k)$. 
Lemma 2.6 Suppose that $k>2$. Then a basis for the coproduct primitive submodule $\mathrm{PH}_{2 k+2} \mathrm{QMO}(k)$ is given by the following set of elements:

$\left\{e_{1} e_{i_{2}} \ldots e_{i_{k}} \mid 1 \leq i_{2} \leq \ldots \leq i_{k}\right\} \cup\left\{e_{2}^{k-2} e_{3}^{2}+e_{1}^{k} \cdot e_{1}^{k-2} e_{2}^{2}, e_{1}^{k-1} e_{2} \cdot e_{1}^{k-1} e_{2}, Q^{k+2} e_{1}^{k}\right\}$.

For $k=1$, a basis for $\mathrm{PH}_{4} Q M O(1)$ is given by

$$
\left\{Q^{3} e_{1}, e_{1} \cdot e_{1} \cdot e_{1} \cdot e_{1}\right\} .
$$

For $k=2$, a basis for $P H_{6} Q M O(2)$ is given by

$$
\left\{e_{1} e_{5}, e_{3}^{2}+e_{1}^{2} \cdot e_{2}^{2}+e_{1}^{2} \cdot e_{1}^{2} \cdot e_{1}^{2}, e_{1} e_{2} \cdot e_{1} e_{2}, Q^{4} e_{1}^{2}\right\} .
$$

Proof Recall that $\psi\left(e_{i}\right)=\sum_{j} e_{j} \otimes e_{i-j}$. This determines $\psi\left(e_{I}\right)$ by naturality. First of all observe that a basis element $e_{i_{1}} e_{i_{2}} \ldots e_{i_{k}}\left(i_{1} \leq i_{2} \leq \ldots \leq i_{k}\right)$ of $H_{*} M O(k)$ is primitive if and only if $i_{1}=1$. To complete the proof we evaluate the coproduct on the non-primitive height 1 basis elements and on all the basis elements of greater height in this dimension.

For $k>2$ there are no basis elements of height greater than 2. For simplicity we use the reduced coproduct $\tilde{\psi}(u)=\psi(u)-u \otimes 1-1 \otimes u$ so that $u$ is primitive when $\tilde{\psi}(u)=0$. Straightforward calculations give the following:

$$
\begin{aligned}
& \tilde{\psi}\left(e_{2}^{k-2} e_{3}^{2}\right)=e_{1}^{k} \otimes e_{1}^{k-2} e_{2}^{2}+e_{1}^{k-2} e_{2}^{2} \otimes e_{1}^{k} \\
& \tilde{\psi}\left(e_{2}^{k-1} e_{4}\right)=e_{1}^{k} \otimes e_{1}^{k-1} e_{3}+e_{1}^{k-1} e_{2} \otimes e_{1}^{k-1} e_{2}+e_{1}^{k-1} e_{3} \otimes e_{1}^{k} ; \\
& \tilde{\psi}\left(e_{1}^{k} \cdot e_{1}^{k-1} e_{3}\right)=e_{1}^{k} \otimes e_{1}^{k-1} e_{3}+e_{1}^{k-1} e_{3} \otimes e_{1}^{k} ; \\
& \tilde{\psi}\left(e_{1}^{k} \cdot e_{1}^{k-2} e_{2}^{2}\right)=e_{1}^{k} \otimes e_{1}^{k-2} e_{2}^{2}+e_{1}^{k-2} e_{2}^{2} \otimes e_{1}^{k} ; \\
& \tilde{\psi}\left(e_{1}^{k-1} e_{2} \cdot e_{1}^{k-1} e_{2}\right)=0 ; \\
& \tilde{\psi}\left(Q^{k+2} e_{1}^{k}\right)=0 .
\end{aligned}
$$

The lemma follows immediately from these results.

Similar calculations give the results for $k=1$ and $k=2$.

This lemma has the following immediate corollary.

Corollary 2.7 For $k>1$, a basis for the projection of the coproduct primitive submodule $h_{*}^{2} \mathrm{PH}_{2 k+2} Q M O(k) \subseteq H_{2 k+2} D_{2} M O(k)$ is given by the following set of elements:

$$
\left\{e_{1}^{k} \cdot e_{1}^{k-2} e_{2}^{2}, e_{1}^{k-1} e_{2} \cdot e_{1}^{k-1} e_{2}, Q^{k+2} e_{1}^{k}\right\} .
$$

For $k=1$ a basis for $h_{*}^{2} P H_{4} Q M O(1)$ is given by $\left\{Q^{3} e_{1}\right\}$.

To complete the proof of the main theorem it is convenient to consider various cases depending on the value of $k$ modulo 4 . 


\section{Case 1: $k$ even}

Theorem 3.1 For even $k$, the double point self-intersection surface of each self-transverse immersion $f: M^{k+2} \rightarrow \mathbb{R}^{2 k+2}$ has even Euler characteristic and so is a boundary.

To prove this result we evaluate dual Steenrod operations on the elements given by Corollary 2.7.

Lemma 3.2 For even $k$, we have the following results in $H_{*} D_{2} M O(k)$ :

$$
\begin{aligned}
& S q_{*}^{1}\left(e_{1}^{k} \cdot e_{1}^{k-2} e_{2}^{2}\right)=S q_{*}^{1}\left(e_{1}^{k-1} e_{2} \cdot e_{1}^{k-1} e_{2}\right)=0 ; \\
& S q_{*}^{1}\left(Q^{k+2} e_{1}^{k}\right)=Q^{k+1} e_{1}^{k} ; \\
& S q_{*}^{2}\left(e_{1}^{k} \cdot e_{1}^{k-2} e_{2}^{2}\right)=S q_{*}^{2}\left(e_{1}^{k-1} e_{2} \cdot e_{1}^{k-1} e_{2}\right)=e_{1}^{k} \cdot e_{1}^{k} .
\end{aligned}
$$

Proof These are immediate from the Steenrod squares in $B O(1)=\mathbb{R} P^{\infty}$ $\left(\mathrm{Sq}_{*}^{i} e_{j}=\left(\begin{array}{c}j-i \\ i\end{array}\right) e_{j-i}\right)$ and the Nishida relations (see [17]).

Corollary 3.3 For even $k$, given $\alpha \in \pi_{2 k+2}^{S} M O(k)$,

$$
h_{*}^{2} h(\alpha)=\lambda\left(e_{1}^{k} \cdot e_{1}^{k-2} e_{2}^{2}+e_{1}^{k-1} e_{2} \cdot e_{1}^{k-1} e_{2}\right)
$$

for $\lambda \in \mathbb{Z} / 2$.

Proof By Lemma 2.5(a) $h_{*}^{2} h(\alpha)$ is a linear combination of the elements given by Corollary 2.7. But since, by Lemma $2.5(\mathrm{~b})$, it is annihiliated by $\mathrm{Sq}_{*}^{1}$ and $\mathrm{Sq}_{*}^{2}$ only elements of the given form can arise.

Proof of Theorem 3.1 Suppose that $f$ corresponds to $\alpha \in \pi_{2 k+2} Q M O(k)$. Then

$$
\xi_{*} h_{*}^{2} h(\alpha)=\lambda \xi_{*}\left(e_{1}^{k} \cdot e_{1}^{k-2} e_{2}^{2}+e_{1}^{k-1} e_{2} \cdot e_{1}^{k-1} e_{2}\right)=\lambda\left(e_{1}^{2 k-2} e_{2}^{2}+e_{1}^{2 k-2} e_{2}^{2}\right)=0
$$

by Lemma 2.4 .

The result follows by Theorem 2.1 .

We can still ask whether there exists an immersion $f: M^{k+2} \rightarrow \mathbb{R}^{2 k+2}$ corresponding to $\alpha \in \pi_{2 k+2}^{S} M O(k)$ with $h_{*}^{2} h(\alpha)=e_{1}^{k} \cdot e_{1}^{k-2} e_{2}^{2}+e_{1}^{k-1} e_{2} \cdot e_{1}^{k-1} e_{2}$. To answer this we first observe the following result. 
Proposition 3.4 For even $k$, given an immersion $f: M^{k+2} \rightarrow \mathbb{R}^{2 k+2}$ corresponding to $\alpha \in \pi_{2 k+2}^{S} M O(k)$, the Hurewicz image $h(\alpha) \in H_{2 k+2} Q M O(k)$ is determined by the bordism class of $M$.

Proof Suppose that $k>2$. Notice that the height 1 part of $h(\alpha)$ is given by $h^{S}(\alpha) \in H_{2 k+2} M O(k) \subseteq H_{2 k+2} Q M O(k)$ (see Diagram (1) in [2]) and so

$$
h(\alpha)=h^{S}(\alpha)+h_{*}^{2} h(\alpha)
$$

using the obvious inclusion $H_{*} D_{2} M O(k) \subseteq H_{*} Q M O(k)$ as the elements of height 2. Furthermore, $h^{S}(\alpha)$ is determined by the normal Stiefel-Whitney numbers of $M$ and so by the bordism class of $M$ (see [2] Lemma 2.2).

Suppose that $f_{1}: M_{1}^{k+2} \rightarrow \mathbb{R}^{2 k+2}$ and $f_{2}: M_{2}^{k+2} \rightarrow \mathbb{R}^{2 k+2}$ are two immersions of bordant manifolds $M_{1}$ and $M_{2}$ corresponding to $\alpha_{1}, \alpha_{2} \in \pi_{2 k+2}^{S} M O(k)$ respectively. Since $M_{1}$ and $M_{2}$ are bordant manifolds $h^{S}\left(\alpha_{1}\right)=h^{S}\left(\alpha_{2}\right)$. It follows that

$$
\begin{aligned}
h\left(\alpha_{1}\right)-h\left(\alpha_{2}\right) & =h_{*}^{2} h\left(\alpha_{1}\right)-h_{*}^{2} h\left(\alpha_{2}\right) \\
& =h_{*}^{2} h\left(\alpha_{1}-\alpha_{2}\right) \\
& =\lambda\left(e_{1}^{k} \cdot e_{1}^{k-2} e_{2}^{2}+e_{1}^{k-1} e_{2} \cdot e_{1}^{k-1} e_{2}\right)
\end{aligned}
$$

by Corollary 3.3. However $e_{1}^{k} \cdot e_{1}^{k-2} e_{2}^{2}+e_{1}^{k-1} e_{2} \cdot e_{1}^{k-1} e_{2} \in H_{2 k+2} Q M O(k)$ is not primitive (by Lemma 2.6) and so not spherical.

Hence $h\left(\alpha_{1}\right)-h\left(\alpha_{2}\right)=0$ and so $h\left(\alpha_{1}\right)=h\left(\alpha_{2}\right)$ as required.

For $k=2$ the above proof has to be modified to take account of height 3 elements. This leads in this case to

$$
h\left(\alpha_{1}\right)-h\left(\alpha_{2}\right)=\lambda\left(e_{1}^{2} \cdot e_{2}^{2}+e_{1} e_{2} \cdot e_{1} e_{2}+e_{1}^{2} \cdot e_{1}^{2} \cdot e_{1}^{2}\right)
$$

which gives the same result.

From now on we write $\alpha(m)$ for the number of digits 1 in the dyadic expression for the positive integer $m$.

Proposition 3.5 For even $k>2$, given an immersion $f: M^{k+2} \rightarrow \mathbb{R}^{2 k+2}$ corresponding to $\alpha \in \pi_{2 k+2}^{S} M O(k)$, the Hurewicz image $h(\alpha) \in H_{2 k+2} Q M O(k)$ is given as follows:

$$
h(\alpha)=\left\{\begin{array}{l}
h^{S}(\alpha), \quad \text { if the normal Stiefel-Whitney number } \bar{w}_{2} \bar{w}_{k}[M]=0, \\
h^{S}(\alpha)+e_{1}^{k} \cdot e_{1}^{k-2} e_{2}^{2}+e_{1}^{k-1} e_{2} \cdot e_{1}^{k-1} e_{2}, \quad \text { if } \bar{w}_{2} \bar{w}_{k}[M]=1 .
\end{array}\right.
$$

The second case arises if and only if $\alpha(k+2) \leq 2$. In this case, writing $k+2=2^{r}+2^{s}$ where $r \geq s \geq 1$, we can take $M$ to be $\mathbb{R} P^{2^{r}} \times \mathbb{R} P^{2^{s}}$. 
For $k=2$, given $f: M^{4} \rightarrow \mathbb{R}^{6}$ corresponding to $\alpha \in \pi_{6}^{S} M O(2)$, the Hurewicz image $h(\alpha) \in H_{6} Q M O(2)$ is given as follows:

$$
h(\alpha)=\left\{\begin{array}{l}
0, \quad \text { if } \bar{w}_{2}^{2}[M]=0, \\
e_{3}^{2}+e_{1}^{2} \cdot e_{2}^{2}+e_{1} e_{2} \cdot e_{1} e_{2}+e_{1}^{2} \cdot e_{1}^{2} \cdot e_{1}^{2}, \quad \text { if } \bar{w}_{2}^{2}[M]=1 .
\end{array}\right.
$$

Proof Suppose that $k>2$ and that $f: M^{k+2} \rightarrow \mathbb{R}^{2 k+2}$ is an immersion corresponding to $\alpha \in \pi_{2 k+2}^{S} M O(k)$.

If $\lambda=0$ in Corollary 3.3 then $h(\alpha)=h^{S}(\alpha)$. By Lemma 2.6, $\lambda=1$ if and only if $e_{2}^{k-2} e_{3}^{2}$ has coefficient 1 when $h^{S}(\alpha)$ is written in terms of the basis $\left\{e_{I}\right\}$. This occurs if and only if the Stiefel-Whitney number $\bar{w}_{2} \bar{w}_{k}[M]=1$. For, by [2] Lemma 2.2, $\bar{w}_{2} \bar{w}_{k}[M]$ is given by the Kronecker product $\left\langle w_{2} w_{k}^{2}, h^{S}(\alpha)\right\rangle$ in $M O(k)$. By naturality, this product can be evaluated in $B O(1)^{k}$ using $\mu_{k}$ and the fact that $\mu_{k}^{*} w_{i}=\sigma_{i}\left(x_{1}, x_{2}, \ldots, x_{k}\right) \in \mathbb{Z} / 2\left[x_{1}, x_{2}, \ldots x_{k}\right] \equiv H^{*} B O(1)^{k}$, the $i$ th elementary symmetric polynomial. In this case $\mu_{k}^{*}\left(w_{2} w_{k}^{2}\right)=\sum x_{1}^{3} x_{2}^{3} x_{3}^{2} \ldots x_{k}^{2}$ and so the Kronecker product $\left\langle w_{2} w_{k}^{2}, h^{S}(\alpha)\right\rangle$ is given by the coefficient of $e_{2}^{k-2} e_{3}^{2}$. Hence $\lambda=1$ if and only if $\bar{w}_{2} \bar{w}_{k}[M]=1$.

In the case $\alpha(k+2)>2$, by a theorem of RLW Brown ([6] Theorem 5.1), there exists an embedding $f_{1}: M_{1} \hookrightarrow \mathbb{R}^{2 k+2}$ of a manifold $M_{1}$ bordant to $M$. Suppose that $f_{1}$ corresponds to $\alpha_{1} \in \pi_{2 k+2}^{S} M O(k)$. Then $h_{*}^{2} h\left(\alpha_{1}\right)=0$ since $f_{1}$ is an embedding with no double points. Hence, by Proposition $3.4, h_{*}^{2} h(\alpha)=0$ and so $h(\alpha)=h^{S}(\alpha)$.

For $\alpha(k+2) \leq 2$ put $k+2=2^{r}+2^{s}$ where $r \geq s \geq 1$. Then by Whitney's immersion theorem there are immersions $\mathbb{R} P^{2^{r}} \rightarrow \rightarrow \mathbb{R}^{2^{r+1}-1}$ and $\mathbb{R} P^{2^{s}} \rightarrow \mathbb{R}^{2^{s+1}-1}$ and the product of these gives an immersion $f: \mathbb{R} P^{2^{r}} \times \mathbb{R} P^{2^{s}} \leftrightarrow \mathbb{R}^{2^{r+1}+2^{s+1}-2}=$ $\mathbb{R}^{2 k+2}$. Finally a standard verification shows that the normal Stiefel-Whitney number $\bar{w}_{2} \bar{w}_{2^{r}+2^{s}-2}\left[\mathbb{R} P^{2^{r}} \times \mathbb{R} P^{2^{s}}\right]=1$.

The argument for $k=2$ is almost identical. The manifold $M^{4}$ either is a boundary or is bordant to $\mathbb{R} P^{2} \times \mathbb{R} P^{2}$ depending on the value of the normal Stiefel-Whitney number $\bar{w}_{2}^{2}[M]$. The presence of the height 3 term $e_{1}^{2} \cdot e_{1}^{2} \cdot e_{1}^{2}$ in the Hurewicz image shows that in this case any immersion of a non-boundary has an odd number of triple points.

\section{$4 \quad$ Case 2: $k \equiv 1 \bmod 4$}

Theorem 4.1 For $k \equiv 1 \bmod 4$, given any manifold $M^{k+2}$ there exists an immersion $M^{k+2} \leftrightarrow \mathbb{R}^{2 k+2}$ with double point self-intersection surface of odd 
Euler characteristic (and so a non-boundary) and there exists another immersion with double point self-intersection surface of even Euler characteristic (and so a boundary).

Proof For $k \equiv 1 \bmod 4, \alpha(k+2) \geq 2$ and so there exists an immersion $f: M^{k+2} \rightarrow \mathbb{R}^{2 k+2}$ by $\mathrm{R}$ Cohen's immersion theorem ([7]). Furthermore, for such $k$ there is an immersion $S^{k+2} \rightarrow \mathbb{R}^{2 k+2}$ with double point manifold of odd Euler characteristic ([13] Theorem 1.2). Taking the connected sum of this immersion and $f$ gives an immersion of $M$ with a double point self-intersection surface with Euler characteristic of opposite parity to that of the double point self-intersection surface of $f$. Hence both parities can arise.

Again it is natural to ask about the Hurewicz image $h(\alpha) \in H_{2 k+2} M O(k)$ of the elements $\alpha \in \pi_{2 k+2}^{S} M O(k)$ corresponding to these immersions. The situation is the following.

Proposition 4.2 For $k \equiv 1 \bmod 4$ such that $k>1$, given an immersion $f: M^{k+2} \leftrightarrow \rightarrow \mathbb{R}^{2 k+2}$ corresponding to $\alpha \in \pi_{2 k+2}^{S} M O(k)$, then the Hurewicz image $h(\alpha) \in H_{2 k+2} Q M O(k)$ is given as follows:

$$
h(\alpha)=\left\{\begin{array}{l}
h^{S}(\alpha), \quad \text { if } \Delta_{2}(f) \text { has even Euler characteristic, } \\
h^{S}(\alpha)+Q^{k+2} e_{1}^{k}, \quad \text { if } \Delta_{2}(f) \text { has odd Euler characteristic. }
\end{array}\right.
$$

For $k=1$, given an immersion $f: M^{3} \rightarrow \mathbb{R}^{4}$ corresponding to $\alpha \in \pi_{4}^{S} M O(1)$, then the Hurewicz image $h(\alpha) \in H_{4} Q M O(1)$ is given as follows:

$$
h(\alpha)=\left\{\begin{array}{l}
0, \quad \text { if } \Delta_{2}(f) \text { has even Euler characteristic, } \\
Q^{3} e_{1}+e_{1} \cdot e_{1} \cdot e_{1} \cdot e_{1}, \quad \text { if } \Delta_{2}(f) \text { has odd Euler characteristic. }
\end{array}\right.
$$

Proof Evaluating the dual Steenrod operations on the elements of Corollary 2.7 we obtain

$$
\begin{aligned}
& \operatorname{Sq}_{*}^{1}\left(e_{1}^{k} \cdot e_{1}^{k-2} e_{2}^{2}\right)=\operatorname{Sq}_{*}^{1}\left(e_{1}^{k-1} e_{2} \cdot e_{1}^{k-1} e_{2}\right)=\operatorname{Sq}_{*}^{1}\left(Q^{k+2} e_{1}^{k}\right)=0 \\
& \operatorname{Sq}_{*}^{2}\left(e_{1}^{k} \cdot e_{1}^{k-2} e_{2}^{2}\right)=\operatorname{Sq}_{*}^{2}\left(e_{1}^{k-1} e_{2} \cdot e_{1}^{k-1} e_{2}\right)=e_{1}^{k} \cdot e_{1}^{k} \\
& \operatorname{Sq}_{*}^{2}\left(Q^{k+2} e_{1}^{k}\right)=0 .
\end{aligned}
$$

Hence $h_{*}^{2} h(\alpha)$ is in the submodule spanned by the set

$$
\left\{e_{1}^{k} \cdot e_{1}^{k-2} e_{2}^{2}+e_{1}^{k-1} e_{2} \cdot e_{1}^{k-1} e_{2}, Q^{k+2} e_{1}^{k}\right\} .
$$

For $k \equiv 1 \bmod 4$ and $k>1$ it follows that $\alpha(k+2)>2$; so, by R L W Brown's embedding theorem ([6] Theorem 5.1), $M$ is bordant to a manifold $M_{1}$ which 
has an embedding $f_{1}: M_{1} \hookrightarrow \mathbb{R}^{2 k+2}$. Let $f_{1}$ correspond to $\alpha_{1} \in \pi_{2 k+2}^{S} M O(k)$. Then $h_{*}^{2} h\left(\alpha_{1}\right)=0$ since $f_{1}$ is an embedding. Furthermore, $h^{S}(\alpha)=h^{S}\left(\alpha_{1}\right)$ since these are determined by the bordism class. It follows, by Lemma 2.6, that $h_{*}^{2} h(\alpha)=h\left(\alpha-\alpha_{1}\right)=\lambda Q^{k+2} e_{1}^{k}$ since this is necessarily a primitive class in $H_{2 k+2} Q M O(k)$. Hence $h(\alpha)=h^{S}(\alpha)+\lambda Q^{k+2} e_{1}^{k}$ where, by Theorem 2.1 and Lemma 2.4, $\lambda$ gives the parity of the Euler characteristic of the double point self-intersection surface of the immersion.

For $k=1$, given any immersion $f: M^{3} \rightarrow \mathbb{R}^{4}$ the manifold $M$ is necessarily a boundary since this is true of all 3 -manifolds and so, if $\alpha \in \pi_{4}^{S} M O(1) \cong$ $\mathbb{Z} / 2$ is the corresponding element, $h^{S}(\alpha)=0$. The formula for $h(\alpha)$ then follows from the value for the Hurewicz image coming from [10] Proposition 3.4. The presence of the height 4 term $e_{1} \cdot e_{1} \cdot e_{1} \cdot e_{1}$ when $\Delta_{2}(f)$ has odd Euler characteristic indicates that such immersions have an odd number of quadruple points.

\section{$5 \quad$ Case 3: $k \equiv 3 \bmod 4$}

Theorem 5.1 For $k \equiv 3 \bmod 4$, there exists a manifold $M^{k+2}$ with a selftransverse immersion $M^{k+2} \rightarrow \mathbb{R}^{2 k+2}$ for which the Euler characteristic of the double point self-intersection surface is odd (so that it is a non-boundary) if and only if $k+1$ is a power of 2 . In this case, the parity of the Euler characteristic of the double point self-intersection surface of a self-transverse immersion $M^{k+2} \rightarrow \mathbb{R}^{2 k+2}$ is given by the normal Stiefel-Whitney number $\bar{w}_{2} \bar{w}_{k}[M]$.

To prove this we begin as in Case 1 .

Lemma 5.2 For $k \equiv 3 \bmod 4$, we have the following results in $H_{*} D_{2} M O(k)$ :

$$
\begin{aligned}
& S q_{*}^{1}\left(e_{1}^{k} \cdot e_{1}^{k-2} e_{2}^{2}\right)=S q_{*}^{1}\left(e_{1}^{k-1} e_{2} \cdot e_{1}^{k-1} e_{2}\right)=S q_{*}^{1}\left(Q^{k+2} e_{1}^{k}\right)=0 \\
& S q_{*}^{2}\left(e_{1}^{k} \cdot e_{1}^{k-2} e_{2}^{2}\right)=S q_{*}^{2}\left(e_{1}^{k-1} e_{2} \cdot e_{1}^{k-1} e_{2}\right)=S q_{*}^{2}\left(Q^{k+2} e_{1}^{k}\right)=e_{1}^{k} \cdot e_{1}^{k} .
\end{aligned}
$$

Corollary 5.3 For $k \equiv 3 \bmod 4$, given $\alpha \in \pi_{2 k+2}^{S} M O(k), h_{*}^{2} h(\alpha)$ lies in the submodule of $\mathrm{H}_{2 k+2} \mathrm{D}_{2} \mathrm{MO}(k)$ spanned by the following set:

$$
\left\{e_{1}^{k} \cdot e_{1}^{k-2} e_{2}^{2}+e_{1}^{k-1} e_{2} \cdot e_{1}^{k-1} e_{2}, e_{1}^{k} \cdot e_{1}^{k-2} e_{2}^{2}+Q^{k+2} e_{1}^{k},\right\} .
$$

To prove Theorem 5.1 we determine which of the elements in the submodule of Corollary 5.3 can arise as a value of $h_{*}^{2} h$. First of all we observe that, for 
$\alpha \in \pi_{2 k+2}^{S} M O(k)$ corresponding to a self-transverse immersion $f: M^{k+2} \rightarrow$ $\mathbb{R}^{2 k+2}$, the value of $h_{*}^{2} h(\alpha)$ and so the bordism class of the double point selfintersection surface is determined by the bordism class of $M$. This is the analogue of Proposition 3.4.

Proposition 5.4 For $k \equiv 3 \bmod 4$, given an immersion $f: M^{k+2} \rightarrow \mathbb{R}^{2 k+2}$ corresponding to $\alpha \in \pi_{2 k+2}^{S} M O(k)$, the Hurewicz image $h(\alpha) \in H_{2 k+2} Q M O(k)$ is determined by the bordism class of $M$.

Before proving this we use it to complete the proof of Theorem 5.1 for $k+1$ not a power of 2 .

Corollary 5.5 For $k \equiv 3 \bmod 4$, given a self-transverse immersion $f: M^{k+2} \uparrow \rightarrow$ $\mathbb{R}^{2 k+2}$, the bordism class of $\Delta_{2}(f)$, the double point self-intersection surface, is determined by the bordism class of $M$.

Proof This is immediate from the preceding proposition since, by Theorem 2.1, the bordism class of $\Delta_{2}(f)$ is determined by $h_{*}^{2} h(\alpha)$.

Theorem 5.1 for $k+1$ not a power of 2 is an immediate consequence of this.

Corollary 5.6 For $k \equiv 3 \bmod 4$ such that $\alpha(k+2)>2$, the double point self-intersection surface of each self-transverse immersion $f: M^{k+2} \rightarrow \mathbb{R}^{2 k+2}$ has even Euler characteristic and so is a boundary.

Proof By R L W Brown's embedding theorem ([6] Theorem 5.1), $M$ is bordant to a manifold $M_{1}$ which has an embedding $f_{1}: M_{1} \hookrightarrow \mathbb{R}^{2 k+2}$. The double point self-intersection surface of $f_{1}$ is empty. Hence, by Corollary 5.5, the double point self-intersection surface of $f$ is a boundary.

We shall return to Theorem 5.1 for $k$ such that $\alpha(k+2)=2$, ie, $k+1$ is a power of 2, after proving Proposition 5.4.

Proof of Proposition 5.4 Suppose that $f_{1}: M_{1}^{k+2} \rightarrow \mathbb{R}^{2 k+2}$ and $f_{2}: M_{2}^{k+2} \rightarrow$ $\mathbb{R}^{2 k+2}$ are two immersions of bordant manifolds corresponding to $\alpha_{1}, \alpha_{2} \in$ $\pi_{2 k+2}^{S} M O(k)$ respectively. As in the proof of Proposition 3.4, since $M_{1}$ and $M_{2}$ are bordant manifolds $h^{S}\left(\alpha_{1}\right)=h^{S}\left(\alpha_{2}\right)$. It follows that

$$
h\left(\alpha_{1}-\alpha_{2}\right)=h\left(\alpha_{1}\right)-h\left(\alpha_{2}\right)=h_{*}^{2} h\left(\alpha_{1}\right)-h_{*}^{2} h\left(\alpha_{2}\right)=h_{*}^{2} h\left(\alpha_{1}-\alpha_{2}\right)
$$


and so lies in the submodule of $H_{2 k+2} Q M O(k)$ spanned by the set given by Corollary 5.3.

We consider the non-zero elements of this submodule in turn. First of all, by Lemma 2.6, neither $e_{1}^{k} \cdot e_{1}^{k-2} e_{2}^{2}+e_{1}^{k-1} e_{2} \cdot e_{1}^{k-1} e_{2}$ nor $e_{1}^{k} \cdot e_{1}^{k-2} e_{2}^{2}+Q^{k+2} e_{1}^{k}$ is primitive and so neither of these elements can be spherical. This leaves the element $e_{1}^{k-1} e_{2} \cdot e_{1}^{k-1} e_{2}+Q^{k+2} e_{1}^{k}$, which is primitive.

Lemma 5.7 The element $e_{1}^{k-1} e_{2} \cdot e_{1}^{k-1} e_{2}+Q^{k+2} e_{1}^{k} \in H_{2 k+2} Q M O(k)$ is not spherical.

Accepting this for the moment, it follows that $h\left(\alpha_{1}\right)-h\left(\alpha_{2}\right)=h\left(\alpha_{1}-\alpha_{2}\right)=0$ and so $h\left(\alpha_{1}\right)=h\left(\alpha_{2}\right)$ as required.

The proof of Lemma 5.7 needs an additional idea. Certain elements $\alpha \in$ $\pi_{n} Q X \cong \pi_{n}^{S} X$ are detected by their Hurewicz image in $H_{n} Q X$. Elements may also be detected by a cohomology operation in $H^{*} C_{\alpha}$ where the stable space $C_{\alpha}$ is the mapping cone of the stable map $\alpha: S^{n} \rightarrow X$. It is interesting to consider the relationship between these two approaches. One of the most basic facts is the following.

Proposition 5.8 Given $\alpha \in \pi_{2 m} Q X \cong \pi_{2 m}^{S} X$, if $h(\alpha)=u^{2} \in H_{2 m} Q X$ where $u \in H_{m} X$, then $S q^{m+1} \bar{u} \neq 0 \in H^{2 m+1} C_{\alpha}$, where $\bar{u} \in H^{m} C_{\alpha}$ is any class such that the Kronecker product $\left\langle i^{*} \bar{u}, u\right\rangle=1$ writing $i: X \rightarrow C_{\alpha}$ for the natural inclusion map.

Notice that $i^{*} \mathrm{Sq}^{m+1} \bar{u}=\mathrm{Sq}^{m+1} i^{*} \bar{u}=0 \in H^{2 m+1} X$ for dimensional reasons and so $\mathrm{Sq}^{m+1} \bar{u}$ lies in $p^{*} H^{2 m+1} S^{2 m+1} \cong \mathbb{Z} / 2$ where $p: C_{\alpha} \rightarrow S^{2 m+1}$ is the natural projection map. When $\mathrm{Sq}^{m+1} \bar{u} \neq 0$ the stable space $C_{\alpha}$ requires at least one suspension before it can exist as an unstable space.

Proof of Proposition 5.8 This proposition can be thought of as describing how detection by a certain James-Hopf invariant corresponds to detection by a Steenrod-Hopf invariant. The proposition follows from work of Boardman and Steer ([5] Corollary 5.15). The special case of $X=\mathbb{R} P^{\infty}$ appears as [11] Lemma 4.2 and the justification given there extends to the general case.

Proof of Lemma 5.7 Suppose, for contradiction, that there exists an element $\alpha \in \pi_{2 k+2} Q M O(k)$ such that

$$
h(\alpha)=e_{1}^{k-1} e_{2} \cdot e_{1}^{k-1} e_{2}+Q^{k+2} e_{1}^{k} \in H_{2 k+2} Q M O(k) .
$$


Adjointness gives a natural isomorphism $\sigma: \pi_{n} Q X \cong \pi_{n+1} Q \Sigma X$. Since the corresponding homology suspension $\sigma: H_{n} Q X \rightarrow H_{n+1} Q \Sigma X$ kills Pontrjagin products and commutes with the Kudo-Araki operations,

$$
h\left(\sigma^{2} \alpha\right)=Q^{k+2} \sigma^{2} e_{1}^{k}=\sigma^{2} e_{1}^{k} \cdot \sigma^{2} e_{1}^{k} \in H_{2 k+4} Q \Sigma^{2} M O(k) .
$$

Hence, by Proposition 5.8,

$$
\mathrm{Sq}^{k+3} \bar{u} \neq 0 \in H^{2 k+5} C_{\sigma^{2} \alpha}
$$

where $\bar{u} \in H^{k+2} C_{\sigma^{2} \alpha}$ is an element such that $\left\langle i^{*} \bar{u}, \sigma^{2} e_{1}^{k}\right\rangle=1$.

We now show that this is impossible.

First of all notice that, since $h^{S}\left(\sigma^{2} \alpha\right)=0 \in H_{2 k+4} \Sigma^{2} M O(k), i^{*}: H^{i} C_{\sigma^{2} \alpha} \cong$ $H^{i} \Sigma^{2} M O(k)$ for $i \neq 2 k+5$. This means that away from dimension $2 k+5$ we can do calculations in $H^{*} C_{\sigma^{2} \alpha}$ by calculating in $H^{*} \Sigma^{2} M O(2)$. In particular, $H^{k+2} C_{\sigma^{2} \alpha} \cong H^{k+2} \Sigma^{2} M O(k) \cong \mathbb{Z} / 2$ is generated by $\sigma^{2} w_{k}$ and so $\bar{u}=\sigma^{2} w_{k}$. Here $w_{k} \in H^{k} M O(k) \subseteq H^{k} B O(k)$ is the universal Stiefel-Whitney class.

Secondly, we can evaluate the action of the Steenrod squaring operations on $H^{*} \Sigma^{2} M O(k)$ using the fact that the operations commute with suspension and using their action on $H^{*} M O(k)$ which is determined by the $\mathrm{Wu}$ formula (see [18] Problem 8A). In evaluating the operations we use the fact that $k \equiv 3 \bmod 4$ which means that we can write $k=4 r-1$ for some positive integer $r$.

Then,

$$
\begin{aligned}
\mathrm{Sq}^{k+3} \bar{u} & =\mathrm{Sq}^{4 r+2} \sigma^{2} w_{4 r-1} \\
& \left.=\mathrm{Sq}^{2} \mathrm{Sq}^{4 r}+\mathrm{Sq}^{1} \mathrm{Sq}^{4 r} \mathrm{Sq}^{1}\right) \sigma^{2} w_{4 r-1} \quad \text { by the Adem relations } \\
& =\mathrm{Sq}^{2} \sigma^{2} \mathrm{Sq}^{4 r} w_{4 r-1}+\mathrm{Sq}^{1} \sigma^{2} \mathrm{Sq}^{4 r} \mathrm{Sq}^{1} w_{4 r-1} \\
& \quad \text { since the operations commute with suspension } \\
= & 0+\mathrm{Sq}^{1} \sigma^{2} w_{1}^{2} w_{4 r-1}^{2} \quad \text { by the Wu formula and dimension } \\
= & \mathrm{Sq}^{1} \sigma^{2} \mathrm{Sq}^{1} w_{1} w_{4 r-1}^{2} \quad \text { by a further application of the Wu formula } \\
= & \mathrm{Sq}^{1} \mathrm{Sq}^{1} \sigma^{2} w_{1} w_{4 r-1}^{2} \quad \text { since } \mathrm{Sq}^{1} \text { commutes with suspension } \\
= & 0 \quad \text { by the Adem relations. }
\end{aligned}
$$

This contradicts equation (3) and so proves the lemma, completing the proof of Proposition 5.4 and so the proof of Theorem 5.1 in the case of $\alpha(k+2)>2$.

Now suppose that $k \equiv 3 \bmod 4$ and $\alpha(k+2)=2$. In this case $k+1=2^{r}$ for some integer $r$ such that $r \geq 2$.

To complete the proof of Theorem 5.1 we consider the structure of the bordism group $\mathrm{MO}_{k+2}$ of $(k+2)$-dimensional manifolds. Recall that the unoriented 
bordism ring $M O_{*}$ is a polynomial ring with one generator in each dimension not of the form $2^{s}-1$. We describe a manifold $M^{k+2}$ as decomposable (up to bordism) if it represents a decomposable element in this ring and so is bordant to a union of product manifolds $N_{1}^{m_{1}} \times N_{2}^{m_{2}}$ where $m_{1}, m_{2}>0$. We can assume that $m_{1}, m_{2} \geq 2$ since there is no non-boundary in dimension 1 . Any indecomposable manifold of dimension $k+2$ can be taken as a representative of a polynomial generator in this dimension and any two such manifolds are bordant modulo decomposable manifolds. Notice that, by R Cohen's immersion theorem $([7])$, every manifold in this dimension immerses in $\mathbb{R}^{2 k+2}$.

We first of all deal with the decomposable manifolds.

Proposition 5.9 For $k \equiv 3 \bmod 4$ such that $\alpha(k+2)=2$, every manifold $M^{k+2}$ which is decomposable in the bordism ring is bordant to a manifold which embeds in $\mathbb{R}^{2 k+2}$. Hence the double point self-intersection surface of any immersion $M^{k+2} \rightarrow \mathbb{R}^{2 k+2}$ has even Euler characteristic and so is a boundary.

Proof To prove that decomposable manifolds of dimension $k+2$ embed up to bordism it is sufficient to prove that each product $N_{1}^{m_{1}} \times N_{2}^{m_{2}}$, with $m_{1}, m_{2} \geq 2$ and $m_{1}+m_{2}=k+2$, embeds up to bordism in $\mathbb{R}^{2 k+2}$. To do this we make use of the following result which follows easily from the Whitney embedding theorem.

Lemma 5.10 ([6] Lemma 2.1) If the manifold $N_{1}^{m_{1}}$ immerses in $\mathbb{R}^{s}$, the manifold $N_{2}^{n_{2}}$ embeds in $\mathbb{R}^{t}$ and $s+t \geq 2 m_{1}+1$ then $N_{1} \times N_{2}$ embeds in $\mathbb{R}^{s+t}$.

Since $m_{1}+m_{2}=k+2=2^{r}+1$ and $m_{1}, m_{2} \geq 2$ it follows that $\alpha\left(m_{1}\right) \geq 2$ or $\alpha\left(m_{2}\right) \geq 2$. Suppose without loss of generality that $\alpha\left(m_{2}\right) \geq 2$. Then, by R L W Brown's embedding theorem ([6]), $N_{2}$ is bordant to a manifold which embeds in $\mathbb{R}^{2 m_{2}-1}$. In addition, by the Whitney immersion theorem, $N_{1}$ immerses in $\mathbb{R}^{2 m_{1}-1}$. Hence, by the lemma, $N_{1} \times N_{2}$ is bordant to a manifold which embeds in $\mathbb{R}^{2 m_{1}+2 m_{2}-2}=\mathbb{R}^{2 k+2}$. The numerical condition of the lemma is automatically satisfied: $s+t=2 k+2 \geq 2 m_{1}+1$ since $m_{1} \leq k$.

The final part of the proposition is immediate from Corollary 5.5 as in the proof of Corollary 5.6.

We turn now to the indecomposable manifolds. 
Proposition 5.11 For $k \equiv 3 \bmod 4$ such that $\alpha(k+2)=2$, suppose that $\alpha \in \pi_{2 k+2}^{S} M O(k)$ corresponds to an immersion $f: M^{k+2} \rightarrow \rightarrow \mathbb{R}^{2 k+2}$ of a manifold $M$ which is indecomposable in the bordism ring. Then

$$
h(\alpha)=h^{S}(\alpha)+e_{1}^{k} \cdot e_{1}^{k-2} e_{2}^{2}+Q^{k+2} e_{1}^{k}
$$

and so the double point self-intersection surface $\Delta_{2}(f)$ has odd Euler characteristic and so is not a boundary.

Proof We make use of particular indecomposable manifolds constructed by A Dold ([8]). In dimension $k+2=2^{r}+1$ this manifold $V^{k+2}$ is formed from the product $S^{1} \times \mathbb{C} P^{2^{r-1}}$ by identifying $(u, z)$ with $(-u, \bar{z})$. In Dold's notation this is $P\left(1,2^{r-1}\right)$. He shows that the cohomology ring of $V$ is given by

$$
H^{*} V=\mathbb{Z} / 2[c, d] /\left(c^{2}, d^{2^{r-1}+1}\right)
$$

where $\operatorname{dim}(c)=1$ and $\operatorname{dim}(d)=2$, and the total tangent Stiefel-Whitney class of $V$ is given by

$$
w(V)=(1+c)(1+c+d)^{2^{r-1}+1} .
$$

This implies that the total normal Steifel-Whitney class of $V$ is given by

$$
\bar{w}(V)=(1+c)(1+c+d)^{2^{r-1}-1}
$$

from which it follows that $\bar{w}_{1}(V)=0, \bar{w}_{2}(V)=d$ and $\bar{w}_{k}=\bar{w}_{2^{r}-1}=c d^{2^{r-1}-1}$.

Hence $V$ is an orientable manifold such that the normal Stiefel-Whitney number $\bar{w}_{2} \bar{w}_{k}[V]=1$.

Let $f: V \rightarrow \mathbb{R}^{2 k+2}$ be an immersion corresponding to $\alpha \in \pi_{2 k+2}^{S} M O(k)$. Using the argument in the proof if Proposition 3.5 it follows from the non-vanishing of this Stiefel-Whitney number that $e_{2}^{k-2} e_{3}^{2}$ has coefficient 1 when $h^{S}(\alpha) \in$ $H_{2 k+2} M O(k)$ is written in terms of the basis $\left\{e_{I}\right\}$. Hence from Lemma 2.6 and Corollary 5.3 it follows that either

$$
h(\alpha)=h^{S}(\alpha)+e_{1}^{k} \cdot e_{1}^{k-2} e_{2}^{2}+e_{1}^{k-1} e_{2} \cdot e_{1}^{k-1} e_{2}
$$

or

$$
h(\alpha)=h^{S}(\alpha)+e_{1}^{k} \cdot e_{1}^{k-2} e_{2}^{2}+Q^{k+2} e_{1}^{k}
$$

However, since $V$ is orientable, $h(\alpha)$ lies in the image of $H_{2 k+2} Q M S O(k)$ in $H_{2 k+1} Q M O(k)$. Since $H_{k+1} M S O(k)=0$ the element $e_{1}^{k-1} e_{2}$ does not come from $H_{2 k+1} M S O(k)$ which eliminates the first of the above possibilities. This proves the formula in the proposition for the manifold $V$. However, since any other indecomposable manifold $M$ is bordant to $V$ modulo a decomposable 
manifold the same formula holds for an immersion of $M$ by Proposition 5.4 and Proposition 5.9.

The Euler characteristic of the double point self-intersection surface is now given by Theorem 2.1 since, by Lemma $2.4, \xi_{*}\left(e_{1}^{k} \cdot e_{1}^{k-2} e_{2}^{2}+Q^{k+2} e_{1}^{k}\right)=e_{1}^{2 k-1} e_{3}$.

Proof of Theorem 5.1 Almost everything is given by Corollary 5.6, Proposition 5.9 and Proposition 5.11. The final observation about the Stiefel-Whitney number is clear since, by Lemma $2.6, e_{1}^{k} \cdot e_{1}^{k-2} e_{2}^{2}$ occurs in $h(\alpha)$ if and only if $e_{2}^{k-2} e_{3}^{2}$ does and by the argument in the proof of Proposition 3.5 this is equivalent to $\bar{w}_{2} \bar{w}_{k}[M]=1$.

\section{Comparison with Szücs's results}

To conclude, we compare the results and methods of this paper with those of Szücs in [22]. His methods are more geometric than ours and he gives explicit constructions to show that any surface of even Euler characteristic can occur as the double point self-intersection manifold of a self-tranverse immersion $M^{k+2} \rightarrow \mathbb{R}^{2 k+2}$ if $k$ is even, and any surface can arise if $k \equiv 1 \bmod 4$.

There are two key steps in Szücs's non-existence proof.

Proposition 6.1 ([22] Claim 1) For $k \not \equiv 1 \bmod 4$, the parity of the Euler characteristic of the double point self-intersection surface of a self-transverse immersion $M^{k+2} \rightarrow \mathbb{R}^{2 k+2}$ depends only on the bordism class of $M$.

Proposition 6.2 ([22] Claim 3) For $k \not \equiv 1 \bmod 4$, the Euler characteristic of the double point self-intersection surface of a self-transverse immersion $M^{k+2} \uparrow$ $\mathbb{R}^{2 k+2}$ is even, if $M$ is decomposable in the bordism ring.

These are both included in our results. In fact we have proved a stronger result than Proposition 6.1 as follows.

Proposition 6.3 For $k \not \equiv 1 \bmod 4$, given an element $\alpha \in \pi_{2 k+2}^{S} M O(k)$ corresponding to a self-transverse immersion $M^{k+2} \rightarrow \mathbb{R}^{2 k+2}$, the Hurewicz image $h(\alpha) \in H_{2 k+2} Q M O(k)$ depends only on the bordism class of $M$.

This is our Proposition 3.4 and Proposition 5.4. It implies Proposition 6.1 by Theorem 2.1. 
Szücs's technique for proving Proposition 6.1 is to extend the discussion to include maps which he calls prim maps; these are smooth maps $M^{k+2} \rightarrow \mathbb{R}^{2 k+2}$ which arise as the projection of an immersion $M^{k+2} \rightarrow \mathbb{R}^{2 k+3} \rightarrow \mathbb{R}^{2 k+2}$. Szücs claims that Proposition 6.1 remains true for these maps. However, this must be false since he describes ([22] Lemma 4) how to construct a prim map $f: V^{2^{r}+1} \rightarrow \mathbb{R}^{2^{r+1}}$ of an indecomposable manifold with a double point selfintersection surface of even Euler characteristic, whereas by our Proposition 5.11 the double point self-intersection surface of any immersion of $V$ will have odd Euler characteristic.

Turning to Proposition 6.2 , we have proved this explicitly for $k \equiv 3 \bmod 4$ as Proposition 5.9. It is immediate for even $k$ from Theorem 3.1. It is intriguing that, whereas by our methods the case of even $k$ is the simplest, by Szücs's geometric approach the argument in this case is quite delicate. He readily reduces the question to the case of $\mathbb{R} P^{2^{r}} \times \mathbb{R} P^{2^{s}}$ (using Proposition 6.1 and Brown's embedding theorem as we do) but then dealing with these manifolds calls for some ingenuity. It is not surprising that these manifolds are the difficult ones since these are the even dimensional manifolds for which $h_{*}^{2} h(\alpha)$ is nonzero (by Proposition 3.5).

\section{References}

[1] M A Asadi-Golmankhaneh, Self-intersection manifolds of immersions, $\mathrm{PhD}$ Thesis, University of Manchester (1998)

[2] M A Asadi-Golmankhaneh, P J Eccles, Determining the characteristic numbers of self-intersection manifolds, J. London Math. Soc. (to appear)

[3] T F Banchoff, Triple points and surgery of immersed surfaces, Proc. Amer. Math. Soc. 46 (1974) 407-413

[4] M G Barratt, P J Eccles, $\Gamma^{+}{ }_{-}$structures III: the stable structure of $\Omega^{\infty} \Sigma^{\infty} A$, Topology, 13 (1974) 199-207

[5] J M Boardman, B Steer, On Hopf invariants, Comment. Math. Helv. 42 (1967) 180-221

[6] R L W Brown, Immersions and embeddings up to cobordism, Can. J. Math. 23 (1971) 1102-1115

[7] RL Cohen, The immersion conjecture for differentiable manifolds, Annals of Math. 122 (1985) 237-328

[8] A Dold, Erzeugende der Thomschen Algebra $\mathfrak{R}$, Math Z. 65 (1956) 25-35

[9] E Dyer, R K Lashof, Homology of iterated loop spaces, Amer. J. Math. 84 (1962) 35-88 
[10] P J Eccles, Multiple points of codimension one immersions of oriented manifolds, Math. Proc. Cambridge Philos. Soc. 87 (1980) 213-220

[11] P J Eccles, Codimension one immersions and the Kervaire invariant one problem, Math. Proc. Cambridge Philos. Soc. 90 (1981) 483-493

[12] P J Eccles, Characteristic numbers of immersions and self-intersection manifolds, from: "Proceedings of the Colloquium in Topology, Szekszárd, Hungary, August 1993", Bolyai Society Mathematical Studies 4, James Bolyai Mathematical Society, Budapest (1995) 197-216

[13] P J Eccles, Double point manifolds and immersions of spheres in Euclidean space, from "Proceedings of the William Browder Sixtieth Birthday Conference, March 1994", Annals of Math. Studies 138, Princeton University Press (1997) 125-137

[14] S O Kochman, Bordism, stable homotopy and Adams spectral sequences, American Mathematical Society (1996)

[15] U Koschorke, B Sanderson, Self intersections and higher Hopf invariants, Topology, 17 (1978) 283-290

[16] J Lannes, Sur les immersions de Boy, Lecture Notes in Mathematics 1051, Springer (1984) 263-270

[17] J P May, The homology of $E_{\infty}$ spaces, Lecture Notes in Mathematics 533, Springer (1976) 1-68

[18] J W Milnor, J D Stasheff, Characteristic classes, Ann. of Math. Studies 76, Princeton University Press (1974)

[19] V P Snaith, A stable decomposition of $\Omega^{n} S^{n} X$, J. London Math. Soc. 7 (1974) 577-583

[20] A Szücs, Gruppy kobordizmov l-pogruženǐ I, Acta Math. Acad. Sci. Hungar. 27 (1976) 343-358

[21] A Szücs, Gruppy kobordizmov l-pogruženǐ̌ II, Acta Math. Acad. Sci. Hungar. 28 (1976) 93-102

[22] A Szücs, Double point surfaces of smooth immersions $M^{n} \rightarrow \mathbb{R}^{2 n-2}$, Math. Proc. Cambridge Philos. Soc. 113 (1993) 601-613

[23] P Vogel, Cobordisme d'immersions, Ann. Sci. Ecole Norm. Sup. 7 (1974) 317358

[24] R Wells, Cobordism groups of immersions, Topology, 5 (1966) 281-294 